\title{
Translation and Validation of the HPLP-II From English to Armenian
}

\author{
Zoya Minasyan, $\mathrm{PhD}, \mathrm{MSN}, \mathrm{RN}$, FNP \\ West Coast University, California, United States \\ (iD) https://orcid.org/0000-0002-9557-0860 \\ Leslie C. Hussey, PhD, RN, CNE \\ Walden University, Minnesota, United States \\ (iD) https://orcid.org/0000-0002-7652-2871
}

Contact: zoya.minasyan@gmail.com

\begin{abstract}
As the focus of healthcare shifts toward the social determinants of health, more information about health disparities between different ethnic communities is needed. The Health Promoting Lifestyle Profile II (HPLPII) is an instrument that measures health promotion behavior across various cultural lines. This article describes the translation and validation of the HPLP-II from English to Armenian for the purpose of studying health promotion behavior among the hypertensive and normotensive Armenian American population and addressing health disparities among this community. Translating the HPLP-II required a multistep process to capture cultural linguistic adjustment. First, the researcher, a local bilingual professor, and a professional translation company each created a forward-translation. These three translations were combined by an editor to create one translation with the highest clarity. The researcher then submitted this translation to two bilingual community members for back-translation. A committee composed of the researcher, the community members, and a bilingual healthcare worker reviewed each back-translated item and adjusted those that lost their meanings. Another committee reviewed each item for clarity and consistency. The instrument was then given to five native speakers who were asked to read each question and verbally express what they understood it to be asking. After the researcher made adjustments based on this feedback, the instrument was given to a committee of nine bilingual experts, who reviewed each item and scored it.This multistep, iterative process resulted in an instrument with an item content validity index score of $0.90(>0.78)$ and a scale content validity index of 0.96 (>0.90), and Cronbach's $\alpha=0.936$, suggesting a high level of reliability. The HPLP-II can now be used in future studies regarding health promotion behavior among Armenian Americans.
\end{abstract}

Keywords: health promotion, HPLP II, Armenian, Armenian American, hypertension, health promotion model

Date Submitted: June 9, 2019 | Date Published: September 3, 2019

Recommended Citation

Minasyan, Z., \& Hussey, L. C. (2019). Translation and validation of the HPLP-II from English to Armenian. Journal of Excellence in Nursing and Healthcare Practice, 1, 15-22. https://doi.org/10.5590/JENHP.2019.1.1.03 


\section{Introduction}

As preventable disease rates continue to rise in the United States, public health efforts have shifted their focus to the social determinants of health (Koh, Piotrowski, Kumanyika, \& Fielding, 2011). These include ethnicity, immigration and citizenship status, socioeconomic status, education, employment, family and social support, and community safety (Koh et al., 2011). In part, this approach is meant to eliminate health disparities seen among different ethnic and cultural communities (Koh et al., 2011). Atlhough more research is needed to understand why certain ethnic groups have a higher prevalence of preventable diseases, conducting this research requires reliable translations of instruments that are validated to measure various aspects of health across cultures.

One such instrument is the Health Promoting Lifestyle Profile II (HPLP-II), which measures various dimensions of health promotion behaviors and is based on Nola Pender's health promotion model (HPM; Walker, Sechrist, \& Pender, 1995). The HPLP-II is composed of 52 items across six subscales. These subscales were designed to measure behaviors that were theorized as relevant to a health-promoting lifestyle: Spiritual Growth (i.e., developing optimal health by understanding the meaning, purpose, and goals in one's life; nine items), Interpersonal Relations (i.e., deeper relationships and open communication; nine items), Nutrition (i.e., healthy eating habits; nine items), Physical Activity (i.e., regular engagement in physical activity; eight items), Health Responsibility (i.e., an individual taking responsibility for their own health; nine items), and Stress Management (i.e., using one's own resources to relieve stress and anxiety; eight items; Walker et al., 1995). Items are scored on a Likert-type scale for which $1=$ never, $2=$ sometimes, $3=$ often, and $4=$ routinely. The total score is determined by calculating the mean of the participant's response to each subscale item, which allows for meaningful comparisons across subscales (Walker et al., 1995).

The HPLP-II has been used across cultures and has been successfully translated into many languages, including Persian, Spanish, Japanese, Turkish, Portuguese, Chinese, and others (P. Sousa, Gaspar, Vaz, Gonzaga, \& Dixe, 2015). The HPLP-II is a good general metric of health promotion behaviors because it is easy to use, brief, applicable across ages and cultures, and has already been validated in various cultures both within the United States and globally (P. Sousa et al., 2015).

The HPLP-II was translated into Armenian with permission from the authors and validated for the purposes of a study regarding health promotion behavior among both hypertensive and normotensive Armenian Americans (Minasyan, 2017). Although the translated instrument was used to study hypertension in this population, it can also be used to study a variety of health disparities because it addresses behaviors across the spectrum of health promotion. Adapting this instrument from English to Armenian required not only a direct forward-translation but also a multistep process to capture cultural linguistic adjustment (Carlson, 2000; Mohamadian, Ghannaee, Kortdzanganeh, \& Meihan, 2013; P. Sousa et al., 2015). This article describes the process of translating the instrument and checking the reliability and validity of the translation.

\section{Literature Review}

The theoretical basis for the HPLP-II is Nola Pender's revised HPM (Pender, Murdaugh, \& Parsons, 2015). The HPM does not use fear or intimidation to achieve health promotion behavior, but rather focuses on both the complexities of how individuals move through the world in regards to their personal health and well-being and how they act on and are acted upon by the environment (Pender et al., 2015; Tomey \& Alligood, 2006). Pender's HPM was created and refined for use among vulnerable populations to capture data on groups that experience significant health disparities (Pender et al., 2015). Vulnerable populations include those who do not speak English, ethnic minority groups, and recent immigrants and refugees to the United States (Pender 
et al., 2015). Though not all Armenian Americans are immigrants, refugees, or non-English speakers, they all belong to an ethnic minority and could therefore be subject to health disparities that affect the incidence, prevalence, mortality, and burden of disease among this community (Pender et al., 2015). Development of empowering health promotion programs is essential among vulnerable populations, and the building blocks of those programs are inherent in the HPM's approach.

The HPLP-II has been used successfully across a variety of cultures and has been translated and validated in both short and long forms. The test-retest reliability of the HPLP-II has shown a score of 0.89 , Cronbach's $\alpha$ $=0.94$, indicating very high levels of internal consistency (Walker et al., 1995). Several studies have successfully used a translation of the HPLP-II to study health promotion behavior in minority immigrant communities in the United States (Carlson, 2000; Minasyan, 2017; Sohng, Sohng, \& Yeom, 2002; Tajik, Galvão, \& Siqueira, 2010; Walker, Kerr, Pender, \& Sechrist, 1990). The HPLP-II has also been translated and validated for the purposes of studying various populations within non-English speaking countries (Aghamolaei \& Ghanbarnejad, 2015; Lee \& Loke, 2005; Meihan \& Chung-Ngok, 2011; Mohamadian et al., 2013; P. Sousa et al., 2015; Teng, Yen, \& Fetzer, 2010; Wei et al., 2000; Zeidi, Hajiagha, \& Zeidi, 2012). The Persian version of the HPLP-II eliminated three items to achieve a fit index of 0.93 on the confirmatory factor analysis, and a Cronbach's $\alpha$ of 0.92 for the whole questionnaire (Aghamolaei \& Ghanbarnejad, 2015). The Chinese HPLP-II was validated by an expert panel after a multistep translation process and achieved a content validity index (CVI) of 1, indicating total agreement (Lee \& Loke, 2005). The Taiwanese version of the HPLP-II omitted one item to achieve an acceptable confirmatory factor analysis of $>0.7$, and an acceptable Cronbach's $\alpha$ of 0.73 (Meihan \& Chung-Ngok, 2011). In the Farsi translation, a study was done to translate and validate a 34-question version, which had a cvi of $>0.8$ and a Cronbach's $\alpha$ of 0.86 across all subscales (Mohamadian et al., 2013). All 52 items were retained for the Portuguese (European) version of the HPLP-II, which had a Cronbach's $\alpha$ of 0.925 (P. Sousa et al., 2015). The Japanese version of the HPLP-II returned a Cronbach's $\alpha$ of 0.93, and upon a 2-week retest, it scored 0.91 (Wei et al., 2000). It has been found that this instrument can be very successful across cultures, languages, and geographic locations when carefully translated and validated (P. Sousa et al., 2015).

Forward-translation, that is, directly translating an instrument from one language to another, is not sufficient for creating culturally specific, valid translations (Carlson, 2000; P. Sousa et al., 2015). Creating a valid translation requires achieving equivalence across cultures in the realms of the semantic, conceptual, content, technical, and criterion (P. Sousa et al., 2015). Further, it is important that the results of both the original and translated versions of the instrument are considered equally valid; they cannot be considered equivalent if the results of one are seen as superior in quality to the results of the other (P. Sousa et al., 2015). Although the process of obtaining valid translations is time consuming and expensive, research done with instruments that are poorly translated cannot produce accurate and rigorous results (Carlson, 2000). Therefore, it is vital to the results of research using translated instruments that the translation process be taken as seriously and conducted as carefully as the rest of the data collection process (Carlson, 2000; P. Sousa et al., 2015). Finally, selection of translators is of specific importance (Carlson, 2000). Translators must not only understand both of the languages, but also both of the cultures involved in the instruments (Carlson, 2000). A commercial translation service is not usually adequate for a final product and should instead be used in conjunction with or replaced by an individual with experience in translation and with the two cultures (Carlson, 2000). These details were taken into consideration throughout the process of translation and validation of the HPLP-II from English to Armenian. 


\section{Translation Technique}

Translating and validating the HPLP-II was a multistep process that involved a variety of community and expert participants. Additionally, several steps were needed to create a translation that was both reliable and valid. After obtaining permission from the author to translate the HPLP-II from English to Armenian, the instrument was forward-translated by three separate translators: the researcher, a professional translation service, and a bilingual faculty member of a local community college. The researcher reviewed these three translations for clarity with the bilingual faculty member, then combined them into one, selecting for the clearest language. This working translation was submitted to an Armenian editor who ensured that the content was clear, well structured, and culturally appropriate.

This translation was then given to two experts for back-translation into English. Neither of these experts has previous familiarity with the HPLP-II in any language. Both translators were native English speakers who learned Armenian later (Carlson, 2000). One translator had experience with health concepts as a registered nurse, and the other was familiar with colloquialisms and idiomatic English as a writer and editor for a national magazine. After each of these translators created their own back-translation, the researcher compared them to the original. Items that were unclear after back-translation were revised for clarity and accuracy.

Once a consensus was reached on each item, the translation and back-translation were brought before a committee of four experts, including the researcher, a bilingual healthcare worker, and the two backtranslators from the previous step. This committee went over each question to ensure clarity, consistency, and translation. This committee revised the translation until consensus was reached on each of the 52 items. At this point, the instrument was given to five native Armenian speakers. Each of them was asked to read through each item and then to verbally express what the item was asking (Carlson, 2000; V. D. Sousa \& Rojjanasrirat, 2011). This revealed any inconsistencies between the Armenian translation and the English original, where the verbally expressed item did not match the meaning of the original. As a result, small adjustments were made to the wording of any such questions, and a different native speaker would be asked to read the adjusted item and verbally express the meaning. When no inconsistencies remained, this step was considered complete.

The final step of the translation was to achieve content equivalence validation by nine bilingual experts in the community. Each of these experts was fluent in English and Armenian and was not previously familiar with the HPLP-II. Each was asked to read through the entire survey in both Armenian and English and to then rate each question on a scale of 1 to 4 , for which $1=$ not equivalent, $2=$ unable to assess equivalence, $3=$ equivalent with minor alterations, and $4=$ very equivalent and succinct (Carlson, 2000; V. D. Sousa \& Rojjanasrirat, 2011). Their scores were evaluated in two ways-both by item and by the overall scale validity. The item CVI was assessed by the average score given by all experts, and the average was 0.90, which was greater than or equal to the threshold of 0.78 (Carlson, 2000; V. D. Sousa \& Rojjanasrirat, 2011). The scale CVI was assessed by averaging all of the responses, and was 0.96 , which was greater than or equal to the threshold of 0.90 (Carlson, 2000; V. D. Sousa \& Rojjanasrirat, 2011). No individual items were removed, as none fell below an item CVI score of 0.78 (Lynn, 1986). Finally, the translation was tested for internal consistency using Cronbach's $\alpha$, which returned a score of 0.936, reflecting a high level of internal consistency. 


\section{Discussion}

Health promotion behavior is an important aspect of the morbidity and mortality of any group and gives researchers vital information about the basis of any health disparities (Pender et al., 2015). Socioeconomic barriers have historically created difficulties in research and outreach toward vulnerable populations, including immigrants and racial and ethnic minorities (Koh et al., 2011). To create and foster social change, it is vital to overcome the barriers and bring targeted, culturally specific healthcare to these groups (Pender et al., 2015). The lack of health literacy among vulnerable populations has been associated with worse health status, poorer health knowledge, increased hospitalization, and decreased participation in preventative activities (Pender et al., 2015). Conversely, greater health literacy can result in medical and health decisions that create better health outcomes (Pender et al., 2015). The economic burden of health disparities has been estimated at \$1.24 trillion; if proper health promotion education was provided to these communities along with preventative healthcare, it is likely that this economic burden could be relieved, at least in part (Pender et al., 2015). Without data, it is impossible to know how to effectively target and assist vulnerable populations.

Creating an Armenian version of the HPLP-II was vital to research on the health promotion behavior of hypertensive and normotensive Armenian Americans. This translation can be used for any study that seeks to understand the impact of health promotion behavior on any related phenomenon in the Armenian community. This translation was generated both to make the HPLP-II available to researchers working with Armenian-speaking communities and to provide a tool that can be used in conjunction with acculturation scales for comparative studies across the diaspora. The psychometric properties of the Armenian HPLP-II were satisfactory, showing that it could be used reliably in Armenian-speaking populations living in the United States. Although some studies (Aghmolaei \& Ghanbarnejad, 2015; Mohamadian et al., 2013; Teng et al., 2010) have shown a diminished reliability for the full 52-item instrument in non-Western cultures, and have thus removed various items, this study did not find evidence of this. Therefore, all 52 items were kept. More research is needed to assess the translation's reliability for Armenian-speaking Armenians.

\section{Conclusion}

This translation was conducted as part of a study regarding the health promotion behavior of Armenian Americans who are both hypertensive and normotensive. However, the translated HPLP-II will be useful for any study regarding the health of the Armenian-speaking population. As the HPLP-II continues to be translated across cultures and languages, it will become a vital tool for those engaging with minority communities in the United States, as well as researchers worldwide. 


\section{References}

Aghamolaei, T., \& Ghanbarnejad, A. (2015). Validity and reliablity of the Persian Health-Promoting Lifestyle Profile II Questionnaire. Journal of Research \& Health. 5. 358-365.

Carlson, E. D. (2000). A case study in translation methodology using the Health Promotion Lifestyle Profile II. Public Health Nursing, 17, 61-70. https://doi.org/10.1046/j.1525-1446.2000.00061.x

Koh, H. K., Piotrowski, J. J., Kumanyika, S., \& Fielding, J. E. (2011). Healthy people: A 2020 vision for the social determinants approach. Health Education \& Behavior, 38, 551-557. https://doi.org/10.1177/1090198111428646

Lee, R. L., \& Loke, A. J. Y. (2005). Health-promoting behaviors and psychosocial well-being of university students in Hong Kong. Public Health Nursing, 22, 209-220.

Lynn, M. R. (1986). Determination and quantification of content validity. Nursing Research, 35, 382-385. https://doi.org/10.1097/00006199-198611000-00017

Meihan, L., \& Chung-Ngok, W. (2011). Validation of the psychometric properties of the health-promoting lifestyle profile in a sample of Taiwanese women. Quality of Life Research, 2O, 523-528. https://doi.org/10.1111/j.0737-1209.2005.220304.x

Minasyan, Z. (2017). Health promotion behavior among hypertensive and normotensive Armenian Americans (Doctoral dissertation). School of Nursing, Walden University, Minneapolis, MN. Retrieved from https://scholarworks.waldenu.edu/dissertations/3707/

Mohamadian, H., Ghannaee, M., Kortdzanganeh, J., \& Meihan, L. (2013). Reliability and construct validity of the Iranian version of health-promoting lifestyle profile in a female adolescent population. International Journal of Preventive Medicine, 4, 42. Retrieved from http://ijpm.mui.ac.ir/index.php/ijpm/article/viewFile/664/762

Pender, N. J., Murdaugh, C. L., \& Parsons, M. A. (2015). Health promotion in nursing practice (7th ed.) Harrisonburg, VA: Pearson.

Sohng, K. Y., Sohng, S., \& Yeom, H. A. (2002). Health-promoting behaviors of elderly Korean immigrants in the United States. Public Health Nursing, 19, 294-300. https://doi.org/10.1046/j.15251446.2002.19409.x

Sousa, P., Gaspar, P., Vaz, D. C., Gonzaga, S., \& Dixe, M. A. (2015). Measuring health-promoting behaviors: Cross-cultural validation of the Health-Promoting Lifestyle Profile-II. International Journal of Nursing Knowledge, 26, 54-61. https://doi.org/10.1111/2047-3095.12065

Sousa, V. D., \& Rojjanasrirat, W. (2011). Translation, adaptation and validation of instruments or scales for use in cross-cultural health care research: A clear and user-friendly guideline. Journal of Evaluation in Clinical Practice, 17, 268-274. https://doi.org/10.1111/j.1365-2753.2010.01434.x

Tajik, M., Galvão, H. M., \& Siqueira, C. E. (2010). Health survey instrument development through a community-based participatory research approach: Health promoting lifestyle profile (HPLP-II) and Brazilian immigrants in Greater Boston. Journal of Immigrant and Minority Health, 12, 390-397. https://doi.org/10.1007/s10903-008-9209-4

Teng, H. L., Yen, M., \& Fetzer, S. (2010). Health Promotion Lifestyle Profile-II: Chinese version short form. Journal of Advanced Nursing, 66, 1864-1873. https://doi.org/10.1111/j.1365-2648.2010.05353.x

Tomey, A., \& Alligood, M. (2006). Nursing theorists and their work (6th ed.). St. Louis, MO: Mosby Elsevier. 
Walker, S. N., Kerr, M. J., Pender, N. J., \& Sechrist, K. R. (1990). A Spanish language version of the HealthPromoting Lifestyle Profile. Nursing Research, 39, 268-273. https://doi.org/10.1037/t40087-000

Walker, S. N., Sechrist, K. R., \& Pender, N. J. (1995). Health promotion model-instruments to measure health promoting lifestyle: Health-Promoting Lifestyle Profile (HPLP II) (Adult version). Ann Arbor, MI: University of Michigan, Deep Blue. Retrieved from http://hdl.handle.net/2027.42/85349

Wei, C. N., Yonemitsu, H., Harada, K., Miyakita, T., Omori, S., Miyabayashi, T., \& Ueda, A. (2000). A Japanese language version of the health-promoting lifestyle profile. Nippon Eiseigaku Zasshi (Japanese Journal of Hygiene), 54, 597-606. https://doi.org/10.1265/jjh.54.597

Zeidi, M. I., Hajiagha, A. P., \& Zeidi, B.M. (2012). Reliability and validity of Persian version of the healthpromoting lifestyle profile. Journal of Mazandaran University of Medical Sciences, 21, 102-113. Retrieved from http://jmums.mazums.ac.ir/files/site1/user_files_odobfo/roghiehsafarit-A-10-3774-da86790.pdf 


\section{Contact Hour Information}

\section{Goal Statement}

The goal of this nursing continuing professional development activity is to provide nurses with information about the usefulness of the Armenian translation version of the HPLP-II provides for health education, clinical management, and research in the Armenian-American population.

\section{Outcome Measure}

After completing this nursing continuing professional development activity, nurses will gain knowledge of the usefulness of the Armenian translation version of the HPLP-II provides for health education, clinical management, and research in the Armenian-American population.

\section{Disclosure}

The author and planners of this nursing continuing professional development activity have disclosed no relevant relationships with commercial interests in pertaining to any information within this article.

\section{Contact Hours: 0.4}

Contact Hour Expiration Date: September 3, 2021

\section{How to Obtain Contact Hours for this Activity}

1. Click on the following URL (link) or copy and paste it to Google Chrome or Firefox browser: https://waldenuniversitycne.rievent.com/a/KVIMIO

2. Complete the registration process and respond to the post-test questions (passing score is $80 \%$ ).

3. Complete the evaluation.

4. Print or download and save your certificate.

Walden University is accredited as a provider of nursing continuing professional development by the American Nurses Credentialing Center's Commission on Accreditation. Po469.

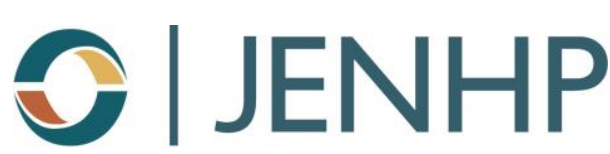

The Journal of Excellence in Nursing and Healthcare

Practice welcomes manuscripts focusing on topics such as national and international workforce issues, quality improvement projects, evidence-based practice initiatives, nursing research studies, interprofessional practice, educational issues, telehealth, improvements in technology, and the impact of social change in society. 